\title{
Aerobic Exercise and S-Klotho Effects on Cardiovascular Disease Patients: A Review
}

\author{
TJ Exford ${ }^{1 *}$, Moran Saghiv², David Ben Sira ${ }^{3}$, Ehud Goldhammer ${ }^{4}$ and Michael Sagiv ${ }^{5}$ \\ ${ }^{1}$ Department of Kinesiology, College of Health and Human Sciences, North Carolina Agriculture \& Technical State University, USA \\ ${ }^{2}$ Department of Kinesiology, College of Health Sciences and Human Performance, University of Texas at Permian Basin, USA \\ ${ }^{3}$ The Academic College at Wingate College, Israel \\ ${ }^{4}$ Heart Institute Bnai-Zion Haifa Medical Center, Technion Institute, Israel \\ ${ }^{5}$ The Academic College at Wingate College, Israel
}

*Corresponding author: TJ Exford, Department of Kinesiology, College of Health and Human Sciences, North Carolina Agriculture \& Technical State University, Greensboro, USA.
Received Date: August 09, 2021

Published Date: August 31, 2021

\begin{abstract}
Coronary artery disease (CAD) is the most common type of heart disease progression once major coronary arteries are injured or diseased. When the primary blood arteries which supply the myocardium with blood, oxygen and nutrients is narrowed arterial disease progresses. Plaque buildup in the walls of the arteries from hypercholesterolemia (high blood cholesterol), dyslipidemia, and vascular inflammation contribute to atherosclerosis formation, a primary agent for CAD. Arterial stiffens occurs as a result of the biological aging process and arteriosclerosis. Endothelial dysfunction is characterized by reduced vascular nitric oxide levels. Nitric oxide vascular reductions leads to irregularities in blood artery function. These functional irregularities result from atherosclerosis, causing vasoconstriction of small arteries. Vasoconstriction of smaller arteries is related to hypertension and could possibly influence, left ventricle diastolic dysfunction. There are two forms of klotho; membrane and secreted, membrane klotho acts as co-receptor for fibroblast growth factor (FGF)-23, while secreted klotho (s-klotho) regulates nitric oxide production in the endothelium minimizing endothelial dysfunction. Studies examining the effect of aerobic exercise on blood circulating s-Klotho have demonstrated a fitness dependent response. S-Klotho values have been shown to be significantly higher in trained vs untrained individuals. Aerobic training is an appropriate model for mechanistically probing the role of physical activity on s-Klotho expression. Factors associated with endothelial function improvement; aerobic fitness levels and aerobic training increased s-klotho levels alleviate and attenuate endothelial dysfunction. Aerobic exercise and klotho gene expression is shown to reduce cardiovascular events in patients with prior CAD thereby decreasing mortality risk.
\end{abstract}

Keywords: Atherosclerosis; Nitric oxide; Reactive oxygen species (ROS); Endothelial dysfunction; Klotho; Coronary artery disease; Oxidative stress

\section{Introduction}

Cardiovascular diseases are prevalent in the general population and are the leading cause of death worldwide responsible for $46.2 \%$ of noncommunicable deaths [1,2]. Coronary artery disease (CAD) broadly comprises CAD myocardial infarction, vascular stiffening, and left ventricular hypertrophy [3]. CAD regularly advances over long periods and is the most common type of heart disease that progresses once major coronary arteries are injured or diseased.
Arterial diseases progress once the main blood arteries that supply the myocardium and the different organs with blood, oxygen, and nutrients is narrowed. Inflammation and cholesterol that creates plaque in the arteries are the cause for arterial diseases [4]. Plaque buildup in the walls of the arteries from hypercholesterolemia, fatty deposits, other substances and inflammatory mechanisms combine dyslipidemia to atheroma development, are usually the main causes 
for CAD [5]. Once plaque is present, decrement in blood flow to the body's organs occur which in turn, decrease blood flow causing reduction in oxygen delivery to the tissues and thus, ischemia [6]. The additional arterial tension and subsequent damage due to hypertension cause the coronary arteries to become narrowed from accumulation of fat, cholesterol and other molecules that collectively is the slow process of chronic inflammatory disease [7]

Atherogenesis is the process of forming plaques in the intima layer of arteries. Plaque's compositions include mainly fat, cholesterol, and calcium [8]. With time, arterial diameter narrows decreasing arterial blood flow. This progression is termed atherosclerosis. Thus, atherogenesis is a multifaceted interface of risk factors with cells of the artery wall, the blood, and molecular communications [9]. Initial atherosclerosis is the consequence of the endothelial cells in the intima layer capturing monocytes along with endothelial permeability aiding the low-density lipoprotein elements to drift into the arterial wall. Myocytes develop and become macrophages that consume the low-density lipoprotein units with apolipoprotein apoB molecule to form foam cells. Oxidative radicals oxidize the $\mathrm{apoB}$ molecule, assemble the units mainly designated to be phagocytized by macrophages [10].

Damage progression is the second stage where the migration of the smooth muscle cells from the arterial wall intima layer into the tunica intima. The next stage is the formation of thrombosis. The rupture of the thin cap fibroatheroma that covers the plaque plus the coagulation components of blood interact with the thrombogenic plaque produces thrombi [11]. In addition, risk factors such as, high cholesterol values, hypertension, diabetes, overweight and oxidative radicals affect the early phase of atherogenesis, namely endothelial dysfunction [12,13]. In brief, atherosclerosis develops progressively with inflammation and lipid accumulation. Atherogenesis is the course of creating plaques in the intima coating of arteries. The buildup of lowdensity lipoprotein and the inflammation of the arterial wall are the first phase of atherosclerosis. Atherogenesis results from lipid peroxidation-derived aldehydes oxidized to carboxylic acids. The pro-inflammatory oxidized phospholipids, resulting of the oxidation of low-density lipoprotein and phospholipids including arachidonic acid, formed in the lipoxygenase and myeloperoxidase pathways, these molecules attract and trigger inflammatory cells, such as monocytes, T-cells, and macrophages. Matrix degradation brings about atherosclerotic as a result of macrophages activation through; cytokines, reactive oxygen species (ROS), and proteolytic enzymes. Oxidative stress is also considered to be a key factor in mechanisms of changes in cell function [14], such as the aging process [15].

Klotho is an anti-aging gene with implications in biological and anatomical processes, mainly in cardiovascular disease [16] Early human aging involving endothelial dysfunction, vascular calcification and progressive atherosclerosis were seen in mice lacking Klotho [17]. In addition, reduced Klotho levels is observed in coronary artery disease patients, physically inactive individuals and in aging. S-Klotho has a role in the action of fibroblast growth factor 23 (FGF23), which directly binds to FGF receptors (FGFRs). This, high affinity complex for FGF23 mediates the intracellular effects of metabolism of phosphorus as the required co-receptor [18]. Other mechanisms which s-klotho is involved, include reserve oxidative stress, inflection of inflammation or attenuation of vascular stiffening $[19,20]$. Therefore, Klotho has been suggested as a master regulator of cardiovascular disease [21].

Aerobic exercise and Klotho gene expression could reduce the risk of cardiovascular events in patients with prior coronary artery disease thus, aerobic exercise may decrease the risk of mortality, incidence and severity of cardiac events [12,15, 22-24]. In addition, in patients with CAD exercise training improves endotheliumdependent vasodilatation both in epicardial coronary vessels and in resistance vessels [24) Patients with significant coronary artery disease present lower soluble concentrations of $\alpha$-Klotho (s-Klotho) [25], as well as reduced levels of Klotho gene expression in the vascular wall [26]. This protein is related to the attenuation of vascular calcification as well as prevention of cardiac hypertrophy [27]. Reduced serum s-Klotho concentrations and decreased vascular Klotho gene expression were associated with the presence as well as the severity of coronary artery disease independently of other established cardiovascular risk factors [14,23]. S-Klotho is a pleiotropic protein related to longevity, which acts as a co-receptor of the fibroblast growth factor 23 and has been proposed as a key regulator of the development of cardiovascular disease. In the few published clinical studies, an association between low levels of s-Klotho and the occurrence and severity of cardiovascular disease have been reported, as well as a reduction of cardiovascular risk when levels were high [28].

\section{Arterial Endothelial Dysfunction}

Arterial endothelium assists vasomotor tone and function by producing and discharging nitric oxide [29]. Arterial endothelium is characterized by cells in the inner layer of all blood arteries and lymphatic system. The inner layer of arteries and veins is the tunica Intima. In arteries, this layer is composed of an elastic membrane lining and smooth endothelium which is a unusual type of epithelial tissue that is covered by elastic tissues. The small thickness of endothelium at the capillaries level permits molecules movements actively and passively as well as ions among blood and lymph by means of the tissues [30]. Following the endothelial layer, elastic fibers thickness varies, which is in a straight line associated to its ability to change vessels' blood volume, pressure and flow velocity [31]. Arterial endothelium contributes to the regulation of blood coagulation, platelet and leukocyte action, vessel's tone and inflammatory responses [32]. The endothelium 
plays an important role in blood vessel functions by producing various signaling molecules [33]. It is a vital regulator of blood flow and blood pressure in the circulatory system. The endothelium is the internal coating of cells that has several critical functions, it controls the correct vessels' radius namely; constriction or dilation, thus changing rapidly blood's amount delivered to the various organs. The endothelium also protects the tissues from various toxic substances by: a. coagulation or formation of a fibrin clot, which is a mechanism that blood changes from a liquid to a gel, it includes activation, bond, and accumulation of platelets along with deposition and maturation of fibrin, b. controls the fluid, c. regulates electrolytes' levels and, d. exchange of numerous substances between the blood and the tissues and Vic versa, and, e. regulates inflammation damages in the tissues. Consequently, Endothelium function is critical for the regular function of the arteries, tissues and organs [34].

Existence of hypertension and dyslipidemia are directly associated to endothelial dysfunction resulting inflammatory and arterial damage [35]. The coronary artery response to acetylcholine rest on the capacity of the endothelium and the endothelial nitric oxide pathway [36]. Arteriosclerosis affects the major elastic and muscular arteries in the extracellular intermediate of arteries elasticity resulting in arterial stiffens [37]. Arterial stiffens outcomes after a deteriorating course affecting mostly the extracellular medium of elastic arteries. It expresses the arterial wall viscoelastic capacity which affects arterial smooth muscle and therefore, the regulation of blood flow, blood pressure, arterial pulse, permeability, and inflammation [38,39]. Changes in extracellular medium proteins and mechanical properties of the arterial wall associated to vessel stiffening can trigger other mechanisms implicated in the progress of atherosclerosis. With time, arterial stiffness increases progressively, exposing the individuals to a greater pressure variation related to increased risk of stroke and renal impairment [40]. Changes in arterial pulsatile lead to an increase in the slow frictional force of blood flow counter to the vessel wall [41]. However, increased wall shear stress reduces nitric oxide production by the endothelial that in turn, increases rate of atheromasias (artery inflammatory) development $[42,43]$. During aerobic exercise training, systolic blood pressure increases thus, pressure in the artery wall rise resulting in the activation of the autoregulation mechanism and thus, vasodilation, that in turn, improves blood flow [44] and thus, has the potential to minimize these pathological process' and to reduce the amount of cardiovascular complications [35].

Dysregulation of the nitric oxide normal smooth muscle contraction properties response is usually characterized by endothelial dysfunction, following advanced atherosclerosis and increase oxidative stress [45]. that induces endothelial dysfunction and atherosclerosis progression by reducing nitric oxide availability [46]. Endothelial dysfunction is a significant moderator in the progress of atherosclerosis and exist long ahead the creation of atherosclerotic plaques [47]. Endothelial dysfunction is mostly triggered by decreased production or action of relaxing mediators. At this point, it is clear that reduced nitric oxide levels cause endothelial dysfunction in blood arterial walls which, leads to vasoconstriction of the artery [48]. and thus, increase blood pressure to high values (hypertension). In addition, it also, activates platelets leading to blood clotting, that increase the stimulation for artery walls inflammation, consequently, arterial walls penetrability to destructive lipoprotein, oxidized free radical species and various toxins is increased.

\section{s-Klotho}

$\alpha$-Klotho protein is found extensively in human tissues of arteries, epithelium, endocrine system, and nerve tissues [49]. Molecular characteristic of Klotho is explained in detail by MartínNunez and colleagues, and therefore it is advised to the reader to look into this article [50]. Klotho appears in two forms; membrane and secreted, the membrane klotho acts as an necessitate coreceptor for fibroblast growth factor (FGF)-23, while secreted klotho regulates nitric oxide production in the endothelium. The extracellular domain of Klotho can be cleaved and cut in the circulation as s-Klotho where it may function as a blood vesselprotective hormone possibly by enhancing endothelial function $[51,52]$ or, direct inhibition of arterial calcification [53]. Circulating s-Klotho is produced in the kidney predominantly expressed in renal distal tubular epithelial cells with implications in biological and anatomical processes, mainly in the cardiovascular arterial's disease [16]. Early human aging that contains endothelial dysfunction, arterial calcification and progressive atherosclerosis were seen in mice lacking Klotho [17]. A reduction in Klotho levels is observed also in aged and physically inactive coronary artery disease patients [26]. These declines are demonstrated by a diminished ability for skeletal muscle to respond to physiological stimuli such as muscle loading or acute injury. Certainly, older adults often exhibit an age-related reduction in the number and size of muscle fibers known as sarcopenia [48]. Klotho appears to apply different functions in distinct cell types as nitric oxidereliant means, Klotho is a putative anti-aging gene, vital cofactor for the linkage of fibroblast growth factor (FGF 23) to its receptor, and thus, acting as a main controller of phosphate balance [18,54]. Studies on mouse genetics have revealed in vivo functions of Klotho (FGF signaling) [55,56].

The Klotho proteins and its connected enzyme $\beta$-Klotho apply various influences on the biological regulation of ion transport, energy metabolism, calcium and phosphate mainly by FGF-23 [57] In addition, s-Klotho functions as an obligate co-receptor with fibroblast growth factor receptor 1 (FGFR1) for fibroblast growth factor 23 (FGF23), a phosphaturic hormone essential for 
maintaining mineral homeostasis [58] Serum FGF-23 values are related to atherosclerotic problem, endothelial dysfunction, arterial stiffness and vascular calcification [59,60] Therefore, Klotho has been suggested as a master regulator of CAD [20,61]. Klotho inhibited the phosphatidyl nitric oxidesitol 3-kinase (PI3K)-AKT signaling pathway phosphorylation of fork head box protein 03a by improving its connection to the manganese superoxide dismutase supporter. Klotho increased mitochondrial manganese superoxide dismutase, mRNA and protein expression. In addition, Klotho reduce tacrolimus-induced oxidative stress and thus, converse mitochondrial dysfunction, consequently, decrease ROS production [56] Increased cellular levels of ROS result in damage to proteins, nucleic acids, lipids, membranes and organelles, which can lead to activation of cell death processes such as apoptosis. Mice with Klotho-deficiency demonstrate an association with accelerated and enhanced development of vasculopathy [62] and early aging. However, increase Klotho expression increase genetic expression that solve the Klotho-deficient phenotype at baseline [16] and thus, improve mice's standing against oxidant stress [63] In humans, serum levels of s-Klotho decrease after 40 years of age $[25,64]$ this may be observed in sedentary individuals and patients with several aging-related diseases such as cardiovascular disease, cancer, hypertension, and kidney disease $[65,66]$.

The clinical relations of s-Klotho and cardiovascular diseases were described earlier $[67,68]$ suggesting that, s-Klotho values in the circulating blood in CAD patients are significantly lower compared to healthy matched peers. In addition, in community-dwelling adults higher plasma Klotho concentrations are independently associated with a lower likelihood of having CAD, heart failure, stroke, or peripheral arterial disease. Recently study by Saghiv, et al. [68] demonstrated that circulating s-Klotho levels are significantly higher while IGF-1 are significantly lower in aerobically trained CAD patients compared to untrained CAD patients and inactive healthy counter partners. Genetic variation studies have demonstrated that Klotho gene polymorphisms might be associated with longevity on one hand [16] and CAD on the other hand [69-71]. Previously Arking, et al. [72] suggested that, the functional of KL-VS allele, characterized by six SNPs is related to hypertension and stroke, and thus, it is a self-regulating risk factor for CAD [73].

Atherosclerosis reduction and cell protection occur by some antioxidant's such as s-Klotho humoral. In addition, arterial stiffening may predispose the intima to atherosclerosis due to injury sustained from increased pulsatile pressure. In mice, klotho through humoral paths guards the arteries by nitric oxide produced in the endothelium [74]. The discovery of Klotho in human vessels tissue $[75,76]$ increased the understanding about the role of the co-expression of two related FGF23 receptors, FGFR-1 and FGFR-3 [76]. Expression of Klotho protein seems to be restricted to arterial intima layer [78]. Yet an argument exists due to inconsistent data regarding Klotho presence in arterial tissue [79]. The connection of Klotho to endothelial dysfunction lays in the attenuation effect on endothelial dysfunction by ways of nitric oxide. Shimada, et al. [80] suggested, that lack of nitric oxide decrease angiogenesis in $\mathrm{kl} / \mathrm{kl}$ mice and reduced endothelium-derived nitric oxide release, due to increased oxidative stress associated with aerobic exercise and aging. Klotho resists oxidative stress by the expression of manganese superoxide dismutase (Mn-SOD) through activation of FoxO forkhead also known as forkhead in rhabdomyosarcoma transcript factor [81] at the cellular and organismal level in mammals. FoxO forkhead is a human protein encoded by the FOXO3 gene [82] FOXO3 belongs to the 0 subclass of the forkhead family of transcription factors which are characterized by a distinct fork head DNA-binding domain [83]. Klotho protein stimulates the FoxO forkhead transcription factors that are negatively regulated by insulin/IGF-1 signaling, so bringing about an expression of manganese superoxide dismutase. With this content, Klotho increases nitric oxide formation through c-AMP-PKA-dependent pathway in human umbilical vascular endothelial cells, [19] and decreases (HO)2-prompt apoptosis and cellular senescence [83].

In addition, Klotho reduces oxidative stress production by limiting angiotensin II production [85] While the complete actions of angiotensin II signaling on NADPH oxidase are still under examination, angiotensin II, is an endogens peptide hormone, that has a major role in maintaining homeostasis in the cardiovascular system, as well as an effective stimulator of NADPH oxidase [86]. Recently Six, et al. [87] observed that attenuation of FGF23 or phosphate-induced vasoconstriction mediated by Klotho is eliminated by adding nitro-L-arginine, a competitive inhibitor of nitric oxide. Moreover, they observed that exposure of human umbilical vein endothelial cells to Klotho increased nitric oxide production and induced nitric oxide phosphorylation and the inducible isoform, nitric oxide, involved in immune response expression. Interestingly, Klotho was able to increase (HO)2 production in cultured human vascular smooth muscle cells, which suggests a more complex effect of this protein on the regulation of vascular tone through mediation of a ROS/ nitric oxide balance [87].

\section{Aerobic Exercise}

Physical inactivity decreases maximal oxygen uptake, muscle mass (sarcopenia), alters structural and intrinsic muscle cells and changes in energy availability which, decrease further with aging $[88,89]$. Cardiac patients in a stage of myocardial deficiency usually lose their muscle mass and muscle strength, due to angiotensin II that directly affects the skeletal muscle and increases protein degradation [90]. Skeletal muscle atrophy is categorized by a reduction in protein contented, fiber width, force generation, and tiredness struggle ability [88]. In addition to angiotensin II, factor such as ROSs cause muscle protein degradation under different situations [88]. The existence of muscle atrophy points out of the 
rise in ROS production and the inability of antioxidant production to balance it resulting, in a reduced protein synthesis [91]. In addition, genetic factors regardless of lifestyle increase rate of biological aging process thereby, severely limiting elderly's function, life quality and longevity [89,92]. However, regardless of age, gender or basal work capacity, aerobic exercise training is recommended as a tool to decrease rate of work capacity decline, typically arises as an individual age, being physically inactive or suffers from any cardiovascular disease [93].

Aerobic exercise was suggested as a nonpharmacological intervention in patients with cardiovascular disease in the main prevention and therapy of cardiovascular diseases [94,95]. It is recommended that exercise intensities be above the anaerobic threshold to vigorous exercise [96]. Yet, exercise mechanisms that slow down atherogenic progress have been completely understood. following long-lasting aerobic exercise, significant changes in the vascular are nitric oxide including decreased levels of C-reactive and inflammatory cytokines [97]. Aerobic exercise has the potential to minimize these pathological process' and to reduce the amount of cardiovascular complications [35]. Effects of chronic aerobic exercise training in CAD and progression of coronary atherosclerosis patients is an increase in myocardial oxygen supply thus, reducing ischemic events. Some indications suggest that long-lasting aerobic exercise may avert loss in endothelium dependent vasodilation and increase levels in sedentary middle to older healthy men. This may represent an important mechanism by which regular aerobic exercise lowers the risk of cardiovascular disease in this population [98]. Aerobic training offers an epigenetic tendency which has benefits in cardiopulmonary and muscular functions, therefore, an individual must interact with environmental factors related to longevity, by exercising at moderate to high level of workloads [99]. In addition, long-lasting aerobic training lessens the decline in maximal oxygen uptake related to physical inactivity and aging [100].

The basic mechanism by which workout triggers genes (epigenetic) includes a stimulus signal to the DNA, then transcript through messenger RNA, and finally conversion into protein [101]. Previously it has been reported that oxidizing free radical species are generated during moderate and high aerobic bouts [102]. ROS production tops cellular defenses, under these conditions, in disease genetic and epigenetic regulation changes gene expression [103]. Skeletal muscle generates superoxide and nitric oxide during aerobic exercise in intensities above the anaerobic threshold [104]. ROS is essential for skeletal muscle force generation, however, ROS in high values may reduce muscle contraction properties and thus, bring about an early exhaustion [105]. There are plentiful indications that exercise can be operative in averting and suspending the result of age on muscle well-being and effectiveness. In addition, chronic exercise training intensifys nitric oxide production, that improves myocardial function due to the increase in coronary blood flow. In addition, nitric oxide has inhibitory effects on platelet and leukocytes well as induces proliferation of arterial smooth fibers [105]. Previous study demonstrate that long-lasting aerobic exercise training reduces cardiovascular risk factors [106]. The nitric oxide synthesis rate seems to be parallel related to increase in amino acid arginine availability [107]. The antithrombotic effect of Aerobic exercise decreases serum levels and activity of inflammatory factors, such as interleukin-6, C-reactive protein, and tumor necrosis factor- $\alpha$, pointing out about the antithrombotic effect of aerobic exercise [108]. Following aerobic bout, blood pressure decreases to lesser levels than those recorded at rest, identified as post exercise hypotension [109]. This autoregulation mechanism response of aerobic exercise coupled with the nitric oxide vasodilator, affects positively the endothelium function and thus blood flow by decreasing total peripheral resistance [110, 111].

Although the association between s-Klotho and aerobic exercise training is not clear, recently, the $\alpha$-Klotho gene is circulating in blood as s-Klotho have been related to the aerobic exercise $[112,113]$. Yet, exercise appears to take a major part on the secreted form of the $\alpha$-Klotho gene in humans. In addition, $\alpha$-Klotho gene is associated also with genes: $\beta$-Klotho gene and $\gamma$-Klotho gene, however, the last two mentioned genes do not have any role during exercise [114]. The increase in s-Klotho following aerobic exercise training may be a response to ROS that increase in muscle cells as a result of aerobic training. $\mathrm{s}$-Klotho reduces apoptosis through the nitric oxide production and thus, suppress oxidative stress [115].

\section{Interplay between Exercise, S-Klotho and Endothelial Dysfunction}

Data suggests that endothelium plays a major part in the regulation of arterial stiffness by the action on smooth muscle tone affected by vasoactive intermediaries and, the effect of nitric oxide production on endothelin arterial stiffness [116]. Nitrogen nitric oxide is a soluble gas molecule with the chemical formula for NO. It is continually synthesized by the endothelium: L-arginine in endothelial cells is the precursor for nitric oxide synthesize by calcium-calmodulin-dependent enzyme nitric oxide synthase [117]. Nitric oxide acts as an endothelium-derived relaxing factor [118]. released from endothelial cells and acts as an inhibitor of ROS production, by decrease in L-arginine endogens asymmetric dimethyl-L-arginine ratio connected with endothelial dysfunction [119]. Nitric oxide stimulates phospholipase A2 and inhibitors of lysolecithin acyltransferase inducing smooth muscle tone relaxation by inhibiting low density lipoprotein oxidation [120]. Klotho has been linked to the prevention of muscle atrophy and cardiovascular disease in aged individuals [14]. Circulating s-Klotho acts as a humoral factor, involved in the endothelium production and regulation of nitric oxide. In turn, nitric oxide protects endothelial 
penetrability, smooth muscles' contraction by calcium homeostasis and inhibits insulin-like growth factor-1 signaling [121]

Similar to Klotho's anti-aging impacts have also been attributed to aerobic exercise [114, 122]. In recent years, there are enough studies regarding the effect of aerobic exercise on blood circulating s-Klotho [23,67] Reimers, et al. [123] demonstrated that the response of s-klotho depends on aerobic fitness level. In addition, levels of s-Klotho were significantly higher in trained individuals compared to untrained once [124]. suggesting that long lasting aerobic training may be an appropriate model for mechanistically probing the role of physical activity on s-Klotho expression. Populations aged 0 - 91 years, screened previously by ELISA revealed that the level of human s-Klotho declines with aging [125]. Previously in older mice, it has been demonstrated that low blood serum s-Klotho levels are related to reduced skeletal muscle strength and aerobic capacity [126]. On the other hand, trained elderly with aerobic capacity have longer life expectancies [127] and higher serum s-Klotho values compared to inactive elderly [112].

Moderate aerobic training attenuates aging-induced pathological cardiac hypertrophy at least partially by restoring s-Klotho levels, reduce oxidative stress, and lessening in the phosphorylation of ERK1/2, P38 and fibrosis [128]. The relationships between aerobic exercise, s-Klotho and endothelial dysfunction can be in brief described as follow: previously it has been suggested that s-Klotho and long-lasting aerobic exercise training are factors that may promote and upgrade young adults' physical performance capacities [129]. Aerobic bout increases s-Klotho levels which in turn, increase FGF23 which promotes nitric oxide synthesis bringing about a, reduction in oxidative stress and ROS in skeletal muscle and accordingly, increase mitochondrial vitality and thus [130] attenuate restore endothelial dysfunction.

\section{Conclusion}

Recent developments point out on the effect of aerobic exercise training program as nitric oxide nonpharmacological mean to support in the treatment, prevention, and therapy of patients with cardiovascular diseases. The present review, suggests that aerobically active CAD patients, increase their Klotho gene expression, which may be a primary involvement to slow down endothelial dysfunction course and cardiovascular-related diseases. In addition, moderate aerobic exercise increase Klotho gene expression in muscle cells and decrease ROD production. Future research should examine the relationship between aerobic exercise training and circulating s-Klotho, on cardiovascular arterial stiffness and endothelial dysfunction.

\section{Acknowledgement}

None.

\section{Conflict of Interest}

No conflict of interest.

\section{References}

1. (2014) Global status report on communicable diseases, Geneva. World Health Organization, Switzerland.

2. Mathers CD, Loncar D (2006) Projections of global mortality and burden of disease from 2002 to 2030. PLoS Med 3: e442.

3. Herzog CA, Asinger RW, Berger AK, (2011) cardiovascular disease in chronic kidney disease. A clinical update from kidney disease: Improving Global Outcomes (KDIGO). Kidney Int 80: 572-586.

4. Hotamisligil GS (2010) Endoplasmic reticulum stress and atherosclerosis Nat Med 16: 396-399.

5. Frostegård J (2010) Low level natural antibodies against phosphorylcholine: a novel risk marker and potential mechanism in atherosclerosis and cardiovascular disease. Clin Immunol 134: 47-54.

6. Fu J, Zou J, Chen C (2008) Hydrogen molecules (H2) improve perfusion recovery via antioxidant effects in experimental peripheral arterial disease. Mol Med Rep 18: 5009-5015.

7. Aziz M, Yadav K (2016) Pathogenesis of atherosclerosis. Med Clin Rev 2: 22. Insull W (2009) The Pathology of atherosclerosis: plaque development and plaque responses to medical treatment. Am J Med 122(1 Suppl): S3-S14.

8. Sun Z (2014) Atherosclerosis and atheroma plaque rupture: normal anatomy of vasa vasorum and their role associated with atherosclerosis. ScientificWorldJournal

9. Sniderman AD, Cianflone K, Arner P (1998) The adipocyte, fatty acid trapping, and atherogenesis. Arterioscler Thromb Vasc Biol 18: 147-151.

10. Sakakura K, Nakanitric oxide M, Otsuka F (2013) Pathophysiology of atherosclerosis plaque progression. Heart Lung Circ 22: 399-411.

11. Hetterich H, Rominger A, Walter L (2016) Natural history of atherosclerotic disease progression as assessed by (18)F-FDG PET/CT Int J Cardiovasc Imaging 32: 49-59.

12. Sargowo D, Ovianti N, Susilowati E (2018) The role of polysaccharide peptide of Ganitric oxidederma lucidum as a potent antioxidant against atherosclerosis in high risk and stable angina patients. Indian Heart J 70: 608-614.

13. Finkel T, Holbrook NJ (2000) Oxidants, oxidative stress and the biology of ageing. Nature 408: 239-247.

14. Frazzia G, Champion HC, Paga PJ (2012) NADPH oxidase-derived ROS and the regulation of pulmonary vessel tone. Am J Physiol Heart Circ Physiol 302: H2166-177.

15. Kuro-o M, Matsumura Y, Aizawa H (1997) Mutation of the mouse klotho gene leads to a syndrome resembling ageing. Nature 390: 45-51.

16. Kuro-o M Klotho (2010) Pflugers Arch 459: 333-343.

17. Kurosu H, Ogawa Y, Miyoshi M (2006) Regulation of fibroblast growth factor-23 signaling by klotho. J Biol Chem 281: 6120-6123.

18. Rakugi H, Matsukawa N, Ishikawa K (2007) Anti-oxidative effect of Klotho on endothelial cells through cAMP activation. Endocrine 31: 8287.

19. Hu MC, Shi M, Zhang J (2011) Klotho deficiency causes vascular calcification in chronic kidney disease. J Am Soc Nephrol. 22: 124-136.

20. Moe SM (2012) Klotho: a master regulator of cardiovascular disease? Circulation 125: 2181-2183.

21. Barouki R (2006) Ageing free radicals and cellular stress. Med Sci (Paris) 22: 266-272.

22. Shirwany AN, Zou NH (2010) Arterial stiffness: a brief review. Acta Pharmacol Sin 31:1267-1276. 
23. Mozos I, Malainer C, Horbańczuk J (2017) Inflammatory markers for arterial stiffness in cardiovascular diseases. Front Immunol 8:1058.

24. Saghiv SM, Ben Sira D, Goldhammer E (2017) The effects of aerobic and anaerobic exercises on circulating soluble-Klotho and IGF-I in young and elderly adults and in CAD patients. J Circ Biomark 6: 1849454417733388

25. Navarro González JF, Donate Correa J, Muros de Fuentes M (2014) Reduced Klotho is associated with the presence and severity of coronary artery disease. Heart 100: 34-40.

26. Yamada S, Giachelli CM (2017) Vascular calcification in CKD-MBD: Roles for phosphate, FGF23, and Klotho. Bone 100: 87-93.

27. Donate Correa J, Martín Nunez E, Mora Fernandez C (2014) Implications of Klotho in vascular health and disease. World J Cardiol 6: 1262-1269.

28. Furchgott RF, Zawadzki JV, Jothiananda D (1980) The obligatory role of endothelial cells in the relaxation of arterial smooth muscle by acetylcholine. Nature 288: 373-376.

29. Gartner LP, Hiatt JL (2013) Color atlas and text of histology. ( $6^{\text {th }}$ edn.), Lippincott. Wiliams \& Wilkins (Ed.), Baltimore, Maryland, US State.

30. Fullstone G, Wood J, Holcombe M (2015) Modelling the Transport of nitric oxide particles under blood flow using an agent-based approach. Sci Rep 5: 10649.

31. Higashi Y, Ueda K, Yoshizumi M (2009) Endothelial function and oxidative stress in cardiovascular diseases. Circ J 73: 411-418.

32. Godo S, Shimokawa H (2017) Divergent roles of endothelial nitric oxide synthases system in maintaining cardiovascular homeostasis. Free Radic Biol Med 109: 4-10.

33. Wang I, Dong X, Gao J (2018) Targeting Inflammatory Vasculature by Extracellular Vesicles. AAPS J 20: 37.

34. De Rosa S, Cirillo P, Paglia A (2010) Reactive oxygen species and antioxidants in the pathophysiology of cardiovascular disease: does the actual knowledge justify a clinical approach? Curr Vasc Pharmacol 8: 259-275.

35. Zeiher AM, Drexler H, Saurbier B, Just H (1993) Endotheliummediated coronary blood flow modulation in humans: effects of age, atherosclerosis, hypercholesterolemia, and hypertension. J Clin Invest 92: 652-662.

36. Palombo C, Kozakova M (2016) Arterial stiffness, atherosclerosis and cardiovascular risk: Pathophysiologic mechanisms and emerging clinical indications. Vascul Pharmacol 77: 1-7.

37. Palatini P, Casiglia E, Gąsowski J (2011) Arterial stiffness, centra hemodynamics, and cardiovascular risk in hypertension. Vasc Health Risk Manag 7: 725-739.

38. Huveneers S, Daemen MJ, Hordijk PL (2015) Between Rho(k) and a hard place: the relation between vessel wall stiffness, endothelial contractility, and cardiovascular disease. Circ Res 116: 895-908.

39. Thorin Trescases N, Thorin E (2016) Lifelong Cyclic Mechanical Strain Promotes Large Elastic Artery Stiffening: Increased Pulse Pressure and Old Age-Related Organ Failure. Can J Cardiol 32: 624-633.

40. Zhao SZ, Ariff B, Long Q (2002) Inter-individual variations in wall shear stress and mechanical stress distributions at the carotid artery bifurcation of healthy humans. J Biomech 35: 1367-1377.

41. Dietz J (2007) Arterial stiffness and extracellular matrix. Adv Cardiol 44: 76-95.

42. Kleinstreuer C, Hyun S, Buchanan JR Jr, PW Longest, JP Archie Jr, et al. (2017) Hemodynamic Parameters and Early Intimal Thickening in Branching Blood Vessels. Crit Rev Biomed Eng 29(1): 1-64.

43. Goto C, Nishioka K, Umemura T, Jitsuiki D, Sakagutchi A, et al. (2007) Acute moderate-intensity exercise induces vasodilation through an increase in nitric oxide bioavailiability in humans. Am J Hypertens 20(8): 825-830.

44. Modaresi A, Nafar M, Sahraei Z (2015) Oxidative stress in chronic kidney disease. Iran J Kidney Dis 9(3): 165-179.
45. Vanhoutte PM, Shimokawa H, Feletou M, EHC Tang, et al. (2017) Endothelial dysfunction and vascular disease - a 30th anniversary update. Acta Physiol (Oxf) 219(1): 22-96.

46. Barthelmes J, Matthias P Nagele, Valeria Ludovici, Frank Ruschitzka, Isabella Sudano, et al. (2017) Endothelial dysfunction in cardiovascular disease and Flammer syndrome-similarities and differences. EPMA J 8(2): 99-109.

47. Avin KG, Coen PM, Huang W, Stolz DB, Sowa GA, et al. (2014) Skeletal muscle as a regulator of the longevity protein, Klotho. Front Physiol 5: 189.

48. Lim K, Groen A, Molostvov G, Lu T, Lilley KS, et al. (2015) $\alpha$-Klotho expression in human tissues. J Clin Endocrinol Metab 100(10): E1308-E1318.

49. Martin-Nunez E, Donate-Correa J, Muros-de-Fuentes M, Mora-Fernández C, Navarro-González JF, et al. (2014) Implications of Klotho in vascular health and disease. World J cardiol 6(12): 1262-1269.

50. Maekawa Y, Ohishi M, Ikushima M, Yamamoto K, Yasuda 0, et al. (2011) Klotho protein diminishes endothelial apoptosis and senescence via a mitogen-activated kinase pathway. Geriatr Gerontol Int 11(4): 510-516.

51. van Ark J, Hammes HP, van Dijk MCRF, Lexis CPH, van der Horst ICC, et al. (2013) Circulating alpha-klotho levels are nitric oxidet disturbed in patients with type 2 diabetes with and without macrovascular disease in the absence of nephropathy. Cardiovasc Diabetol 12: 116-120

52. Semba RD, Cappola AR, Sun K, Bandinelli S, Dalal M, et al. (2011) Plasma klotho and cardiovascular disease in adults. J Am Geriatr Soc 59(9): 1596-1601.

53. Urakawa I, Yamazaki Y, Shimada T, Iijima K, Hasegawaet H, al. (2006) Klotho converts canitric oxidenical FGF receptor into a specific receptor for FGF23. Nature 444(7120): 770-774.

54. Shawkat Razzaque M (2012) The role of Klotho in energy metabolism. Nat Rev Endocrinitric oxidel 8(10): 579-587.

55. Yuechi Xu, Zhongjie Sun (2015) Molecular Basis of Klotho: From Gene to Function in Aging. Endocr Rev 36(2): 174-193.

56. Long YC, Kharitonenkov A (2011) Hormone-like fibroblast growth factors and metabolic regulation. Biochim Biophys Acta 1812(7): 791795.

57. Kuro-o M (2009) Klotho and aging. Biochim Biophys Acta 1790(10): 1049-1058.

58. Mirza MA, Hansen T, Johansson L, Ahlström H, Larsson A, et al. (2009) Relationship between circulating FGF23 and total body atherosclerosis in the community. Nephrol Dial Transplant 24(10): 3125-3131.

59. Mirza MA, Larsson A, Lind L, Larsson TE (2009) Circulating fibroblast growth factor-23 is associated with vascular dysfunction in the community. Atherosclerosis 205(2): 385-390.

60. Donate-Correa J, Martín-Nunez E, Martínez-Sanz R, Muros-de-Fuentes M, Mora-Fernández C, et al. (2016) Polymorphisms on vascular gene expression and its relationship to cardiovascular disease. J Cell Mol Med 20(1): 128-133.

61. Lim SW, Jin L, Luo K (2017) Klotho enhances FoxO -mediated manganese superoxide dismutase expression by negatively regulating $\mathrm{PI}_{3} \mathrm{~K} / \mathrm{AKT}$ pathway during tacrolimus-induced oxidative stress. Cell Death Dis 8(8): e2972.

62. Kurosu H, Yamamoto M, Clark JD, Pastor JV, Nandi A, et al. (2005) Suppression of aging in mice by the hormone Klotho. Science 309(5742): 1829-1833.

63. Sugiura H, Yoshida T, Tsuchiya K, Mitobe M, Nishimura S, et al. (2005) Klotho reduces apoptosis in experimental ischaemic acute renal failure. Nephrol Dial Transplant 20(12): 2636-2645.

64. Matsuda S, Nakagawa Y, Tsuji A, Kitagishi Y, Nakanishi A, et al. (2018) Implications of PI3K/AKT/PTEN Signaling on Superoxide Dismutases Expression and in the Pathogenesis of Alzheimer's Disease. Diseases 6(2): 28. 
65. Wang Y, Sun Z (2009) Current understanding of klotho. Ageing Res Rev 8(1): 43-51.

66. Semba RD, Cappola AR, Sun K, Bandinelli S, Dalal M, et al. (2011) Plasma klotho and mortality risk in older community-dwelling adults. J Gerontol A Biol Sci Med Sci 66(7): 794-800.

67. Saghiv M, Sherve C, Ben Sira D, Sagiv M, Goldhammer E, et al. (2016) Aerobic training effect on blood s-Klotho levels in coronary artery disease patient. J Clin Exp Cardiolog 7(8): 1-4.

68. Imamura A, Okumura K, Ogawa Y, Murakami R, Torigoe M, et al (2006) Klotho gene polymorphism may be a genetic risk factor for atherosclerotic coronary artery disease but not for vasospastic angina in Japanese. Clin Chim Acta 371(1-2): 66-70.

69. Rhee EJ, Oh KW, Lee WY, Kim SY, Jung CH, et al. (2006) The differential effects of age on the association of KLOTHO gene polymorphisms with coronary artery disease. Metabolism 55(10): 1344-1351.

70. Jo SH, Kim SG, Choi YJ, Joo NR, Cho GY, et al. (2009) KLOTHO gene polymorphism is associated with coronary artery stenitric oxidesis but nitric oxidet with coronary calcification in a Korean population. Int Heart J 50(1): 23-32.

71. Arking DE, Becker DM, Yanek LR, Fallin D, Judge DP, et al. (2003) KLOTHO allele status and the risk of early-onset occult coronary artery disease. Am J Hum Genet 72(5): 1154-1161.

72. Arking DE, Atzmon G, Arking A, Barzilai N, Dietz HC, et al. (2005) Association between a functional variant of the KLOTHO gene and highdensity lipoprotein cholesterol, blood pressure, stroke, and longevity. Circ Res 96(4): 412-418.

73. Saito Y, Yamagishi T, Nakamura T, Ohyama Y, Aizawa H, et al. (1998) Klotho protein protects against endothelial dysfunction. Biochem Biophys Res Commun 248(2): 324-329.

74. Lim K, Lu TS, Molostvov G, Lee C, Lam FT, et al. (2012) Vascular Klotho deficiency potentiates the development of human artery calcification and mediates resistance to fibroblast growth factor 23. Circulation 125(18): 2243-2255

75. Donate-Correa J, Mora-Fernández C, Martínez-Sanz R, Muros-de-Fuentes M, Pérez H, et al. (2013) Expression of $\mathrm{FGF}_{23} / \mathrm{KLOTHO}_{\text {system in human }}$ vascular tissue. Int J Cardiol 165(1): 179-183.

76. Cao F, Wang S, Cao X, Liu X, Fu K, et al. (2017) Fibroblast growth factor 21 attenuates calcification of vascular smooth muscle cells in vitro. J Pharm Pharmacol 69(12): 1802-1816.

77. Chang JR, Guo J, Wang Y, Hou YL, Lu WW, et al. (2016) Intermedin1-53 attenuates vascular calcification in rats with chronic kidney disease by upregulation of $\alpha$-Klotho. Kidney Int 89(3): 586-600.

78. Wehling-Henricks M, Welc SS, Samengo G, Rinaldi C, Lindsey C, et at. (2018) Macrophages scape Klotho gene silencing in the mdx mouse model of Duchenne muscular dystrophy and promote muscle growth and increase satellite cell numbers through a Klotho-mediated pathway. Hum Mol Genet 27(1): 14-29.

79. Shimada T, Takeshita Y, Murohara T, Sasaki KI, Egami K, et al. (2004) Angiogenesis and vasculogenesis are impaired in the precocious-aging klotho mouse. Circulation 110(9): 1148-1155.

80. Yamamoto M, Clark JD, Pastor JV, Gurnani P, Nandi A, et al. (2005) Regulation of oxidative stress by the anti-aging hormone klotho. J Biol Chem 280(45): 38029-38034.

81. Anderson MJ, Viars CS, Czekay S, et al. (1998) Cloning and characterization of three human forkhead genes that comprise an FKHRlike gene subfamily. Genitric oxidemics 47(2): 187-199.

82. Greer E, Brunet A (2005) FOXO transcription factors at the interface between longevity and tumor suppression. Oncogene 24(50): 74107425

83. Ikushima M, Rakugi H, Ishikawa K, et al. (2006) Anti-apoptotic and anti-senescence effects of Klotho on vascular endothelial cells. Biochem Biophys Res Commun 339(3): 827-832.
84. Wang Y, Kuro-o M, Sun Z (2012) Klotho gene delivery suppresses Nitric oxidex2 expression and attenuates oxidative stress in rat aortic smooth muscle cells via the cAMP-PKA pathway. Aging Cell 11(3): 410-417.

85. Wen H, Judith K Gwathmey KJ, Xie L-H (2012) Oxidative stress-mediated effects of angiotensin II in the cardiovascular system. World J Hypertens 2: 34-44.

86. Six I, Okazaki H, Gross P, et al. (2014) Direct, acute effects of Klotho and FGF23 on vascular smooth muscle and endothelium. PLoS One 9(4): e93423.

87. Jackman WR, Kandarian CS (2004) The molecular basis of skeletal muscle atrophy. Am J Physiol Cell Physiol 287: C834-C843.

88. Pollock RD, Duggal NA, Lazarus NR, JM Lord, SDR Harridge (2018) Cardiorespiratory fitness nitric oxidet sedentary time or physical activity is associated with cardiometabolic risk in active older adults. Scand J Med Sci Sports 28: 1653-1660.

89. Springer J, Springer JI, Anker S (2017) Muscle wasting and sarcopenia in heart failure and beyond: update 2017. ESC Heart Fail 4: 492-498.

90. Maria Antonietta Pellegrino, Jean-François Desaphy, Lorenza Brocca, Sabata Pierno, Diana Conte Camerino, et al. (2011) Redox homeostasis, oxidative stress and disuse muscle atrophy. J Physiol 589: 2147-2160.

91. Afilalo J, Karunananthan S, Eisenberg MJ, et al. (2009) Role of frailty in patients with cardiovascular disease. Am J Cardiol 103(11): 1616-1621.

92. Nikolaidis PT, Villiger E, Rosemann T, Beat Knechtle (2018) The effect of aging on pacing strategies of cross-country skiers and the role of performance level. Eur Rev Aging Phys Act 15: 4.

93. Billman G (2002) Aerobic exercise conditioning: A nitric oxidenpharmacological antiarrhythmic intervention. J Appl Physiol 92(2): 446-454.

94. Conti AA (2014) Western medical rehabilitation through time: a historical and epistemological review. Scientific World Journal 2014: 432506 .

95. Jennings EG, Dunsiger SI, Bock BC (2018) Setting larger session duration goals is associated with greater future physical activity. PLoS One 13(12): e0208644.

96. Ghadieh SA, Basem Saab (2015) Evidence for exercise training in the management of hypertension in adults. Can Fam Physician 61: 233-239.

97. Virdis A, Ghiadoni L, Giannarelli C, S Taddei (2010) Endothelial dysfunction and vascular disease in later life. Maturitas 67: 20-24.

98. Baldwin KM, Haddad F (2010) Research in the exercise sciences: where we are and where do we go from here--Part II. Exerc Sport Sci Rev 38(2): 42-50.

99. Thomas W Buford, R Gavin MacNeil, Launa G Clough, Marvin Dirain Bhanuprasad Sandesara, et al. (2014) Active muscle regeneration following eccentric contraction-induced injury is similar between healthy young and older adults. J Appl Physiol 116: 1481-1490.

100. Puthucheary Z, Skipworth JR, Rawal J, et al. (2011) Genetic influences in sport and physical performance. Sports Med 41: 845-859.

101. McArdle A, Pattwell D, Vasilaki A, RD Griffiths, MJ Jackson (2001) Contractile activity-induced oxidative stress: cellular origin and adaptive responses. Am J Physiol Cell Physiol 280: C621-627.

102. Afanasev J (2014) New Nucleophilic Mechanisms of Ros-Dependent Epigenetic Modifications: Comparison of Aging and Cancer. Aging Dis 5: 52-62.

103. McArdle A, Pollock N, Staunton CA, Malcolm J Jackson (2018) Aberrant redox signaling and stress response in age-related muscle decline: Role in inter-intra-cellular signaling. Free Radic Biol Med 132: 50-57.

104. Powers KS, Nelson WB, Hudson BM (2011) Exercise-induced oxidative stress in humans: Cause and consequences. Free Radic Biol Med 51: 942-950.

105. Rush JWE, Ford RJ (2007) Nitric oxide, oxidative stress and vascular endothelium in health and hypertension. Clin Hemorh Microc $37(1,2)$ : 185-192. 
106. Duncker DJ, Bache RJ (2008) Regulation of coronary blood flow during exercise. Physiol Rev 88: 1009-1086.

107. Tsung-Han Liu, Ching-Lin Wu, Chi-Wei Chiang, Yu-Wei Lo, Hung$\mathrm{Fu}$ Tseng, et al. (2009) Nitric oxide effect of short-term arginine supplementation on nitric oxide production, metabolism and performance in intermittent exercise in athletes. J Nutr Biochem 20 : 462-468.

108. Petersen BK (2011) Exercise-induced myokines and their role in chronic diseases. Brain Behav Immun 25: 811-816.

109. Brito LC, Fecchio RY, Peçanha T, et al. (2018) Postexercise hypotension as a clinical tool: a "single brick" in the wall. J Am Soc Hypertens 12(12): e59-e64.

110. Hoier B, Hellsten Y (2014) Exercise-induced capillary growth in human skeletal muscle and the dynamics of VEGF. Microcirculation 21 301-314.

111. Steven A Romero, Matthew R Ely, Dylan C Sieck, Meredith J Luttrell, Tahisha M Buck, et al. (2015) Effect of antioxidants on histamine receptor activation and sustained postexercise vasodilatation in humans. Exp Physiol 100: 435-449.

112. Saghiv M, D Ben Sira, Goldhammer E, Sagiv M (2015) Effects of aerobic exercise training on S-Klotho in young and elderly. JJ Physiology 1(1) 001.

113. Amaro-Gahete FJ, Alejandro Dela 0, LucasJurado Fasoli, AndreaEspuch-Oliver, LidiaRobles-Gonzalez, et al. (2018) Exercise training as S-Klotho protein stimulator in sedentary healthy adults: Rationale, design, and methodology. Contemp Clin Trials Commun 18; 11: 10-19.

114. Francisco J Amaro-Gahete, Alejandro de-la-0, Lucas Jurado-Fasoli, Jonatan R Ruiz, Manuel J Castillo, et al. (2018) Role of exercise on S-Klotho protein regulation: a systematic review. Curr Aging Sci 11(2):100-107.

115. Carracedo J, Buendia P, Merinitric oxide A, et al. (2012) Klotho modulates the stress response in human senescent endothelial cells. Mech Ageing Dev 133(11-12): 647-654.

116. Cannon OR (1998) Role of nitric oxide in cardiovascular disease: focus on the endothelium. Clin Chem 44(9): 1809-1819.

117. Kuan-Feng Hsieh, Juey-Ming Shih, Yao-Ming Shih, Man-Hui Pai, SungLing Yeh, et al. (2018) Arginine administration increases circulating endothelial progenitor cells and attenuates tissue injury in a mouse model of hind limb ischemia/reperfusion. Nutrition 56: 29-35.

118. Palmer RM, Ferrige AG, Moncada S (1987) Nitric oxide release accounts for the biological activity of endothelium-derived relaxing factor. Nature 327: 524-526.
119. Engin A (2017) Endothelial Dysfunction in Obesity. Adv Exp Med Biol 960: 345-379.

120. Young SG, Parthasarathy S (1994) Why are low-density lipoproteins atherogenic. West J Med 160(2): 153-164.

121. Masashi Kitagawa, Hitoshi Sugiyama, Hiroshi Morinaga, Tatsuyuki Inoue, Keiichi Takiue, et al. (2013) A decreased level of serum soluble Klotho is an independent biomarker associated with arterial stiffness in patients with chronic kidney disease. PLoS One 8(2): e56695.

122. Nuria Garatachea, Helios Pareja Galeano, Fabian Sanchis-Gomar, Alejandro Santos-Lozano, Carmen Fiuza-Luces, et al. (2015) Exercise attenuates the major hallmarks of aging. Rejuvenation Res 18: 57-89.

123. Reimers CD, Knapp G, Reimers AK (2012) Does physical activity increase life expectancy? A review of the literature. J Aging Res 2012 : 243958.

124. Eun Young Lee, Sang Soo Kim, Ji-Sung Lee, In Joo Kim, Sang Heon Song, et al. (2014) Soluble $\alpha$-Klotho as a Nitric oxidevel Biomarker in the Early Stage of Nephropathy in Patients with Type 2 Diabetes. Plos One 9(8): e102984.

125. Candace L Crasto, Richard D Semba, Kai Sun, Anne R Cappola, Stefania Bandinelli, et al. (2012) Relationship of low-circulating "anti-aging" klotho hormone with disability in activities of daily living among older community-dwelling adults. Rejuvenation Res 15: 295-301.

126. Keith G Avin, Paul M Coen, Wan Huang, Donna B Stolz, Gwendolyn A Sowa, et al. (2014) Skeletal muscle as a regulator of the longevity protein, Klotho. Front Physiol 5: 189.

127. Paffenbarger RS Jr, Kampert JB, Lee IM (1997) Physical activity and health of college men: longitudinal observations. Int J Sports Med 3: S200-203.

128. Baghaiee B, Karimi P, Siahkouhian M, Linda S Pescatello (2018) Moderate aerobic exercise training decreases middle-aged induced pathologic cardiac hypertrophy by improving Klotho expression, MAPK signaling pathway, and oxidative stress status in Wistar rats. Iran J Basic Med Sci 21: 911-919.

129. Saghiv M, Ben-Sira D, Sagiv M (2015) Comparison between aerobic and anaerobic training influence on s-klotho blood levels following $60 \mathrm{~min}$ aerobic bout. JJ Physiology 1(1): 004.

130. Dong-Jie Li, Hui Fu, Ting Zhao, Min Ni, Fu-Ming Shen (2016) Exercisestimulated FGF23 promotes exercise performance via controlling the excess reactive oxygen species production and enhancing mitochondrial function in skeletal muscle Metabolism 65: 747-756. 